\title{
Evaluation of Channels Blacklists in TSCH Networks with Star and Tree Topologies
}

\author{
Diego V. Queiroz \\ Federal University of Campina \\ Grande, Brazil - Universidad \\ Politécnica de Madrid, Spain \\ Campina Grande, Paraíba \\ diego@sti.ufpb.br
}

\author{
Ruan D. Gomes \\ Federal Institute of Education, Science \\ and Technology of Paraíba, Brazil \\ Campina Grande, Paraíba \\ ruan.gomes@ifpb.edu.br
}

\author{
Cesar Benavente-Peces \\ Universidad Politécnica de Madrid \\ Madrid, Spain \\ cesar.benavente@upm.es
}

\author{
Iguatemi E. Fonseca \\ Federal University of Paraíba \\ Joao Pessoa, Brazil \\ iguatemi@ci.ufpb.br
}

\author{
Marcelo S. Alencar \\ Federal University of Bahia \\ Salvador, Bahia, Brazil \\ malencar@dee.ufcg.edu.br
}

\begin{abstract}
The Time-Slotted Channel Hopping (TSCH) mode, defined by the IEEE $802.15 .4 \mathrm{e}$ protocol, aims to reduce the effects of narrowband interference and multipath fading on some channels through the frequency hopping method. To work satisfactorily, this method must be based on the evaluation of the channel quality through which the packets will be transmitted to avoid packet losses. In addition to the estimation, it is necessary to manage channel blacklists, which prevents the sensors from hopping to bad quality channels. The blacklists can be applied locally or globally, and this paper evaluates the use of a local blacklist through simulation of a TSCH network in a simulated harsh industrial environment. This work evaluates two approaches, and both use a developed protocol based on TSCH, called Adaptive Blacklist TSCH (AB-TSCH), that considers beacon packets and includes a link quality estimation with blacklists. The first approach uses the protocol to compare a simple version of TSCH to configurations with different sizes of blacklists in star topology. In this approach, it is possible to analyze the channel adaption method that occurs when the blacklist has 15 channels. The second approach uses the protocol to evaluate blacklists in tree topology, and discusses the inherent problems of this topology. The results show that, when the estimation is performed continuously, a larger blacklist leads to an increase of performance in star topology. In tree topology, due to the simultaneous transmissions among some nodes, the use of smaller blacklist showed better performance.
\end{abstract}

\section{CCS CONCEPTS}

- Networks $\rightarrow$ Network simulations; Link-layer protocols; • Computer systems organization $\rightarrow$ Sensor networks; $\bullet$ Hardware $\rightarrow$ Wireless integrated network sensors;

Permission to make digital or hard copies of all or part of this work for personal or classroom use is granted without fee provided that copies are not made or distributed for profit or commercial advantage and that copies bear this notice and the full citation on the first page. Copyrights for components of this work owned by others than ACM must be honored. Abstracting with credit is permitted. To copy otherwise, or republish, to post on servers or to redistribute to lists, requires prior specific permission and/or a fee. Request permissions from permissions@acm.org.

Q2SWinet'18, October 28-November 2, 2018, Montreal, QC, Canada

(C) 2018 Association for Computing Machinery.

ACM ISBN 978-1-4503-5963-4/18/10 ..\$15.00

https://doi.org/10.1145/3267129.3267131

\section{KEYWORDS}

Time-Slotted Channel Hopping; local blacklist; frequency hopping; simulation

\section{ACM Reference Format:}

Diego V. Queiroz, Ruan D. Gomes, Cesar Benavente-Peces, Iguatemi E. Fonseca, and Marcelo S. Alencar. 2018. Evaluation of channels blacklists in TSCH networks with star and tree topologies. In 14th ACM International Symposium on QoS and Security for Wireless and Mobile Networks (Q2SWinet'18), October 28-November 2, 2018, Montreal, QC, Canada. ACM, New York, NY, USA, 8 pages. https://doi.org/10.1145/3267129.3267131

\section{INTRODUCTION}

The IEEE 802.15.4e standard introduces several changes to the Medium Access Control (MAC) layer if compared to its previous version called IEEE 802.15.4, and five modes of operation (protocols) were defined, of which only three have been effectively researched [10]: TSCH (Time-Slotted Channel Hopping), DSME (Deterministic and Synchronous Multichannel Extension) and LLDN (Low-latency Deterministic Network).

TSCH divides the time into slots and uses the Frequency Hopping Scatter Spectrum (FHSS) to reduce the impact of multipath fading and external interference. This technique requires network synchronization and changes the communication frequency every packet is sent, i.e. in each timeslot, making the network more robust against problems that affect only a subset of the channels. Each timeslot in a TSCH network has an associated channel offset that is converted to a frequency in a pseudorandom hopping method.

A link between two nodes can be represented by a pair of timeslot in the slotframe $(n)$, and the channel offset used by the nodes in that timeslot, defined as [ $n$, channelOffset]. The frequency $f$ to be used to transmit in timeslot $n$ of the slotframe can be calculated as follows:

$$
\left.f=F H S[A S N+\operatorname{chOffset}) \% F H S_{\text {length }}\right],
$$

where ASN (Absolute Slot Number) represents the total number of timeslots incremented in each time interval from the beginning of the network, $\%$ is the modulus operator, and the length of the sequence (FHS_length) is 16 channels by default, without blacklisting in the calculation. 


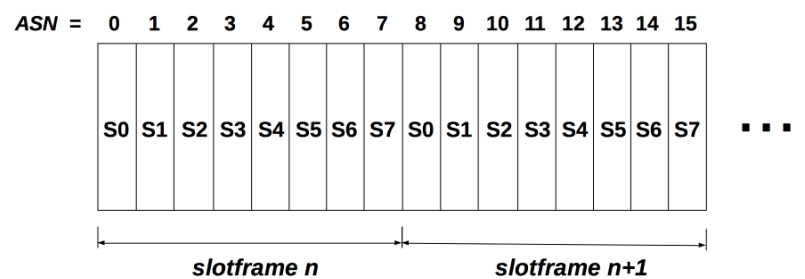

Figure 1: Slotframe with eight timeslots in a TSCH network.

Figure 1 shows an example of slotframe with eight timeslots (S0 to S7) in a TSCH network. Each timeslot lasts $10 \mathrm{~ms}$ like WirelessHART, and allows the transmission of a data packet and the corresponding ACK packet. If a particular transmitter does not receive the ACK packet within the same timeslot, it can retransmit the packet at the next slot allocated to it.

In addition to frequency hopping, TSCH considers the use of blacklists for channels that present low quality. Other standards such as WirelessHART and ISA100.11a, use the concept of frequency hopping and blacklisting as well. WirelessHART employs a central network manager with a frequency hopping and global blacklist approach. ISA100.11a defines a local blacklist scheme, where the nodes abort transmissions on channels that are blacklisted, which may prevent unsuccessful transmissions, but may increase the delay and decrease the Packet Reception Rate (PRR) at the application layer, if packets are dropped before they are transmitted.

There are 16 different channels available for communication; however, due to the inherent problems of wireless networks, especially in industrial environments, some of the channels must be inserted in the blacklist, decreasing the number of the available ones. Because of this, the FHS must be regenerated every time a channel is blacklisted.

The hopping sequences can be of arbitrary length of up to 511 elements and cover all or a subset of available channels for the physical layer [8]. The generation of a new FHS requires the computation of $\mathrm{O}(\mathrm{L})$ complexity, where $\mathrm{L}$ is the length of the FHS. Since $\mathrm{L}$ is generally a large number to ensure randomness $(\mathrm{L}=511$ in the FHS standard), FHS regeneration requires computational resources, which is a problem to be solved.

It is a challenging task to configure the blacklist and to schedule the node transmissions so that two or more neighbor links do not use the same channel at the same time. There are two approaches to creating a blacklist: global and local. In the first approach, all the nodes have the same list of channels, but this solution may become suboptimal, since the channels may present different quality for different network links, even if the nodes are close to each other [6]. In the second method, each pair of nodes can use different sets of channels [9], i.e. a specific blacklist is defined per link. However, local blacklisting mechanisms are more complex than the global approaches, and can introduce collisions when two or more nodes transmit in the same timeslot with different blacklists, which can result in multiple nodes broadcasting on the same channel at the same time. Besides, if the environment is very unstable and the blacklist is modified very often, the local blacklist may not be useful because of too much signaling frames required for resynchronization.

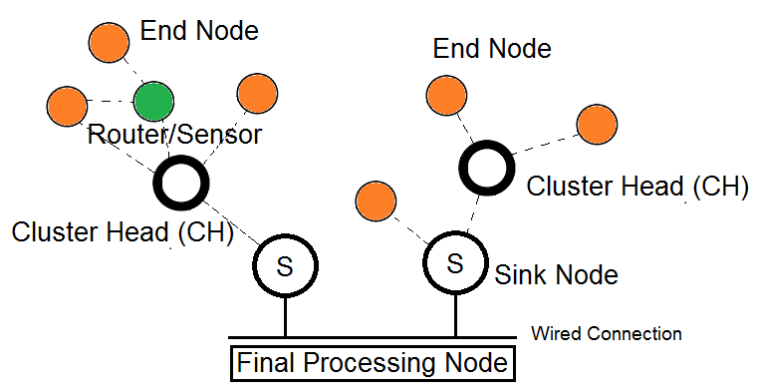

Figure 2: Tree topology network.

In this solution, a dedicated node estimates the quality of the links and decides when the channels need to be blacklisted. This device acts as a link quality estimator node (LQE node) and was described initially in a previous work [6]. Based on the estimation made by the LQE node, AB-TSCH sends the estimation information across the network using signaling frames (enhanced beacons - EB). To evaluate this approach, simulations with different sizes for the blacklist and different topologies were carried out in this work, and a comparison was made with the basic implementation of TSCH, which uses all 16 channels available in all links.

The importance of the correct channel blacklist management in TSCH has been neglected by most of the works, which focus more on scheduling, without worrying about the quality of the channels. This paper addresses this issue and discusses the management challenges.

\section{SCHEDULING AND BLACKLISTS}

When the communication among the nodes is based on beacons, more specifically EBs, each node obtains synchronization, frequency hopping, timeslot and slotframe information in each beacon packet. After receiving an EB, the node is synchronized with the network and initializes the slotframe.

EBs are used to advertise the TSCH network, but the beacons policy is not specified by the IEEE $802.15 .4 \mathrm{e}$ standard. In the proposal presented for AB-TSCH, the coordinator, which also works as sink and link quality estimator node, sends beacons through all the 16 existing channels sequentially. This approach is important because the beacon is not limited to a single channel (usually the first channel), and the nodes that lost synchronization can switch to the other channels to receive those beacons. This reduces the time for a node to be reconnected, increasing the throughput of the network. When the network is built in a tree topology, as depicted in Figure 2, the sink node sends beacons to the cluster heads $(\mathrm{CH})$, which in turn send beacons to the endnodes. In a star topology, only the sink node sends beacons.

The tree topology is more challenging than the star topology because two nodes can send packets simultaneously in the same channel, at the same time (timeslot), causing packet losses. Even if they are in adjacent channels, there is some level of interference that must be mitigated. In star topology, each node is in a different channel and different timeslot, although it is possible to allocate shared slots for many nodes, as depicted in Figure 3. In the latter, there must be some contention-based method to deal with collisions. 
Table 1: Frequency resulting from two nodes with simultaneous transmission, according to Eq. 1.

\begin{tabular}{|c|c|}
\hline ASN & 112 \\
\hline chOffset node 5 & 10 \\
\hline chOffset node 8 & 42 \\
\hline FHS_lenght & 16 \\
\hline Calculated frequency $(f)$ & 10 \\
\hline
\end{tabular}

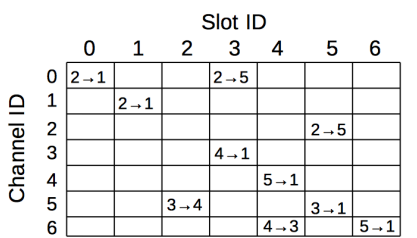

(a)

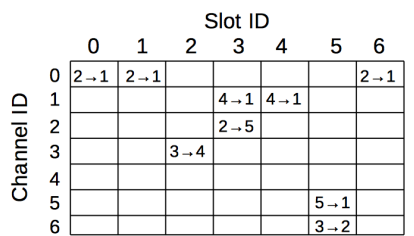

(b)
Figure 3: Channel diversity mechanisms.

In Figure 3 (a), each link between the nodes is allocated in a different channel, and a different timeslot. In Figure 3 (b), the nodes remain in the same channel for a term, and some timeslots are shared for other links, as in the third slot $(4 \rightarrow 1$ and $2 \rightarrow 5)$. The first approach (a) is called channel hopping, which is the default for TSCH and optional for the DSME mode, and the second one (b) is called channel adaption, and is only used in DSME mode. In [5], there is a discussion about both methods, and the authors propose a third method, a novel hybrid of both. The results showed that the use of channel adaptation is better than channel hopping for the transmission of unicast packets, when the quality of the links are monitored continuously. However, for packets transmitted in broadcast by the coordinator, the use of channel hopping is a good alternative to deal with the spatial variation in the quality of the channels. With 15 channels blacklisted, TSCH mode is "forced" to work with channel adaption method.

In tree topology, after performing the calculation of Eq. 1, it is very likely that some nodes in the same timeslot with different channel offsets to receive the same or adjacent channels, which is a disadvantage since collisions and interference may occur on these communications, as illustrated in Table 1.

The problem with collisions and interference with the blacklist approach in TSCH schedules is addressed by some researchers [2, $4,13]$.

In [2], the authors introduced the Adaptive TSCH (A-TSCH), which uses channel blacklisting. The transmitting nodes become aware of the neighbors' blacklist, and both the transmitter and receiver use the same hopping sequence to communicate by inserting the list information into transmission packets. The same process is performed to maintain the timing information. The A-TSCH was encoded under the protocol stack of the Berkley OpenWSN operating system and implemented on the GINA sensor platform.

In [4], the algorithm MABO-TSCH assigns in a centralized manner a collection of cells for each radio link, while considering local blacklists. Several channel offsets are assigned within the same timeslot, so that a radio link can pick one of the channel offset that does not give a blacklisted physical frequency. To be reactive, a pair of nodes locally decides the channels to be inserted into the list. To maintain a consistent list for the link, they insert it into the ACK package, combined with a sequence number. To estimate channel quality, MABO-TSCH explores the problem of Stochastic Multi-Armed Bandits (MAB).

In [13], the algorithm LOST is presented and relies on information gathered by its 1-hop neighbors only. It multiplexes the different transmissions across different channels by allocating properly the channel offsets. A localized blacklist method is employed in the scheduler to avoid using the bad radio channels.

The dynamic whitelisting method of channels is used in [12]. The authors propose an improved version of TSCH, called Enhanced TSCH (ETSCH), which uses a non-intrusive channel quality estimation technique called NICE. The ETSCH uses the Energy Detection (ED) method of the frequency spectrum to measure the quality of the channels at each time interval. It frequently updates a channel stream and sets up a secondary list with the best channels through which the EBs are transmitted. At least two samples of channel energy are made per time interval, and using a secondary list reduces the likelihood of losing EBs.

\section{SIMULATION MODEL OF INDUSTRIAL WIRELESS SENSOR NETWORKS}

In [7], a simulation model was developed to simulate multi-channel protocols for Industrial Wireless Sensor Networks (IWSN). The model captures the effects of fading, shadowing and non-stationary channel characteristics in industrial environments, as well as considering differences in the behavior and link asymmetry of the 16 channels.

The model was integrated into Castalia [1], an open source simulator, and the Eq. 2 was used to simulate the large-scale path loss and shadowing. It shows the path loss in $\mathrm{dB}$ at distance $d$ between transmitter and receiver,

$$
L(d)=L\left(d_{0}\right)+10 n \log \left(\frac{d}{d_{0}}\right)+X_{\sigma},
$$

where $n$ is the path loss exponent and $d_{0}$ is the reference distance. There is a variation in power received, depending on where the measurement is performed. To capture this variation, a random variable $X \sigma$ included in Eq. 2, where $X \sigma$ is a Gaussian distribution with mean zero and standard deviation $\sigma$, and the two parameters are represented in $\mathrm{dB} . X \sigma$ is known as lognormal shadowing.

In addition to large-scale path loss and shadowing, small-scale attenuation was included in the model, and describes the rapid changes in the multi-path profile of the environment caused by the movement of objects around the receiver and transmitter. By default, the simulator does not implement the characteristics of industrial environments.

In [11], experiments in industrial environments have shown that the distribution of Rice models the time attenuation in such an environment. Rice models the fading on a small scale when there is a dominant stationary signal, and random components overlapping the main component. In industrial environments, there are usually several invariant rays, and only a small part of the multipath profile is affected by moving objects [11]. As described 
Table 2: Wireless channel parameters in the Castalia simulation code with the developed model.

\begin{tabular}{l}
\hline SN.wirelessChannel.pathLossExponent $=1.69$ \\
SN.wirelessChannel.PLd0 $=80.48$ \\
SN.wirelessChannel.d0 $=15$ \\
SN.wirelessChannel.sigma $=6.62$ \\
SN.wirelessChannel.K $=12.3$ \\
SN.wirelessChannel.K_sigma $=5.4$ \\
SN.wirelessChannel.meanTimeChange $=85$ \\
SN.wirelessChannel.seed $=0$
\end{tabular}

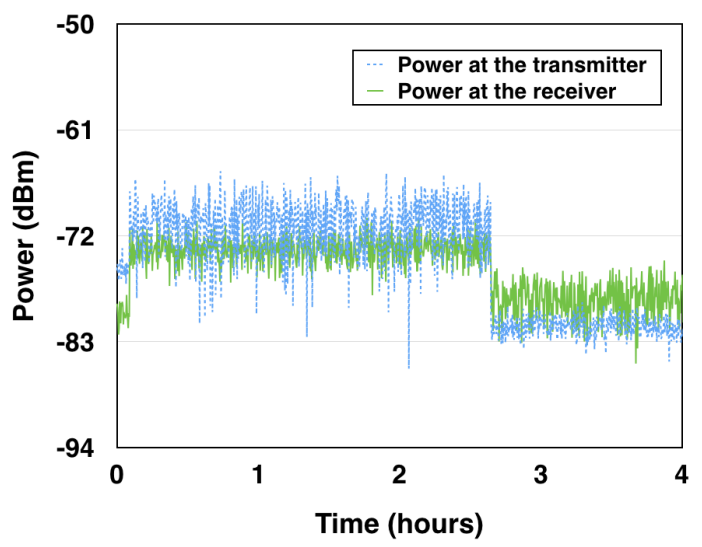

Figure 4: Non-stationary channel characteristics and the asymmetry of the links during the simulation.

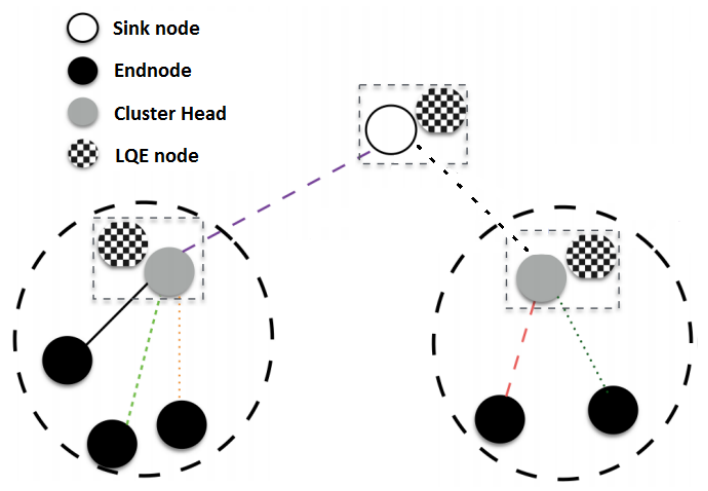

Figure 5: Tree topology with LQE node function integrated into the Cluster Head and Sink nodes.

in [7], this distribution was also implemented in the simulation model, and the wireless channel parameters are defined as indicated in Table 2 in the Castalia simulation script.

Figure 4 illustrates the operation of the simulator in relation to the power reception of packets, and the effects caused by the industrial environments in the network performance.

As a way of monitoring each channel used by the nodes, in this solution the coordinator waits for 10 values of the received signal strength indicator (RSSI) of each data packet to apply the model and obtain an estimate of the PRR of the endnode to the coordinator. If this value falls below the threshold, which has been set to $90 \%$ in the simulated environment, the link may switch its channel at the next multi-slotframe (cycle).

\section{AB-TSCH PROTOCOL}

Following the TSCH standard, in AB-TSCH the ACK packets are sent after reception of a packet in the same timeslot. If one of the nodes does not receive the ACK packet, it considers that the sink node has not received the data packet, and retransmits it in the time interval defined for it in the next cycle. If the sink node has received the original packet in the previous slotframe, but the endnode has not received the ACK, after receiving the retransmitted packet, the sink node considers it as a duplicated packet, and this is accounted for the estimation of channel quality.

To evaluate the blacklist management more quickly, in the simulation the maximum number of retransmissions was set to one, in case of failure on the first attempt, after that the packet is considered lost. However, this parameter is configurable and some authors define even up to eight retransmission packets, as in [3]. In this case, it is necessary to evaluate the dynamics of the network, since a large number of retransmissions decreases its throughput and increases the battery consumption of the nodes to transmit the same packet. In contrast, a small number of retransmissions may generate false positive in relation to the low quality of a given channel. In addition, a study should be made regarding the number of retransmissions and packet transmission rates to achieve high performance. These values depend on several factors; among them are the number of nodes, the capacity of the nodes to process information, the network topology, and the network dynamicity.

Both the cluster and the sink send ACK packets to each other. The endnode, upon receiving the ACK from the cluster head, it considers the packet to be successfully received. The same package is forwarded to the sink by the cluster head, which expects an ACK packet from the sink. If the cluster does not receive the ACK from the sink, it retransmits it in the next cycle.

The method used for managing the blacklist uses a fixed-length blacklist. When a channel becomes poor in quality, according to link quality estimator, it is blacklisted and other blacklisted channel is removed from the list. In this work, some performance metrics were analyzed as the PRR at MAC and application layers, the average number of packet transmission attempts, and latency. The Required Number of Packet transmissions (RNP) is the average number of retries made for each packet generated on the network, regardless of reception. Since two-packet transmission attempts were used, the maximum RNP value in this case is two.

In star topology, the blacklist was first defined statically for the last channels of the network, as depicted in Figure 6 (a). In this example, a blacklist of six channels is initialized, and the nodes cannot send packets to the sink (node 0) on the channels 11-16. During the network execution, after the channel analysis performed by the sink node, each node will have a local blacklist, and sometimes a channel blacklisted at the beginning will be whitelisted after the estimator analysis. In this topology, each node has its own timeslot, 


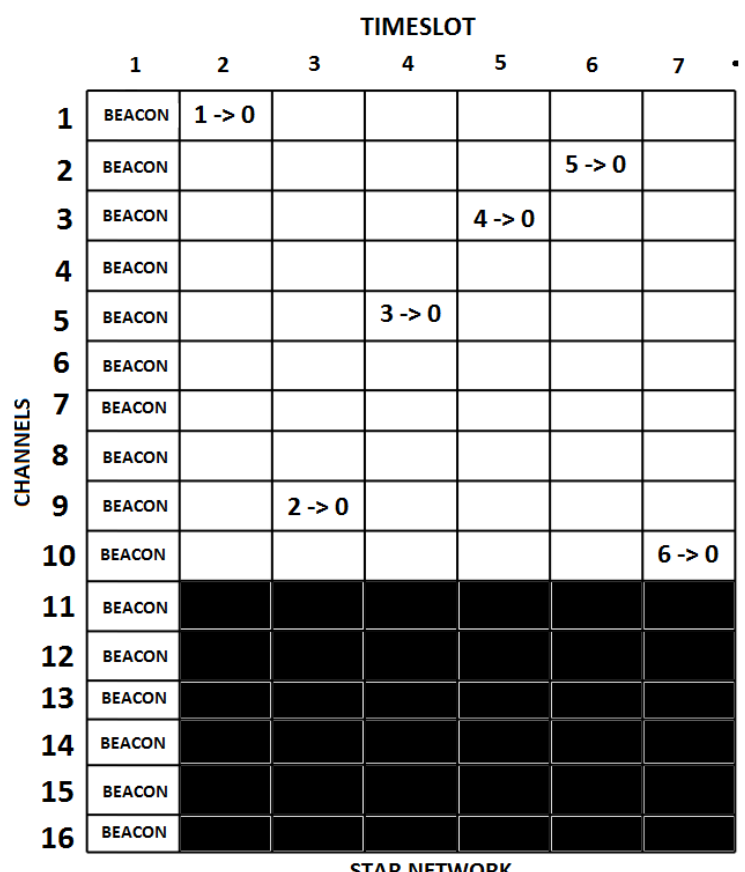

(a)

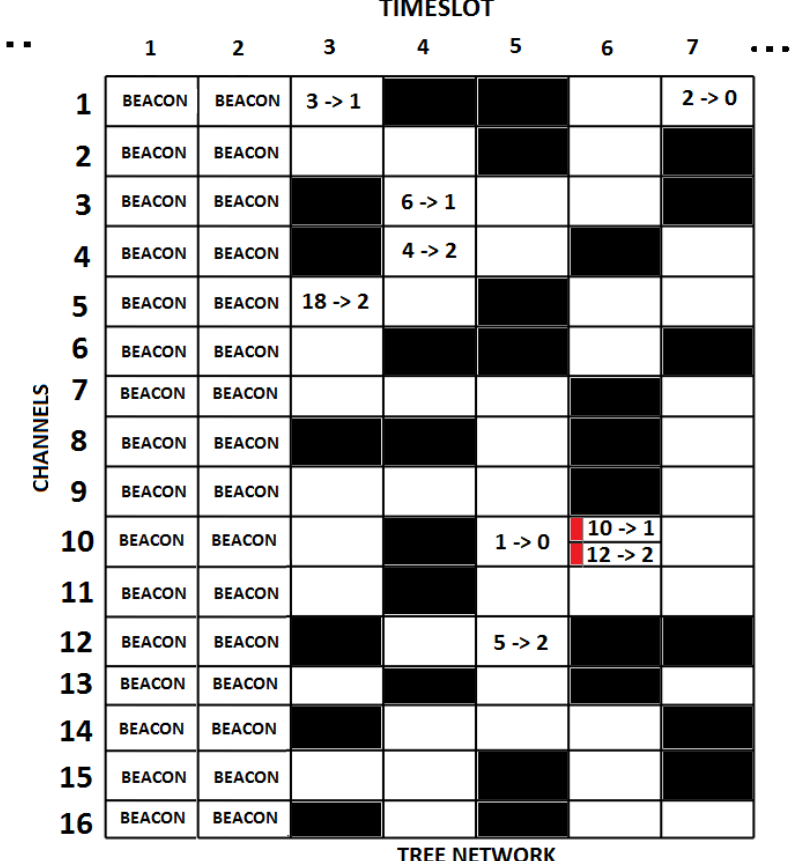

(b)

Figure 6: Example of schedule and blacklists of six channels for the experiment in star and tree topologies.

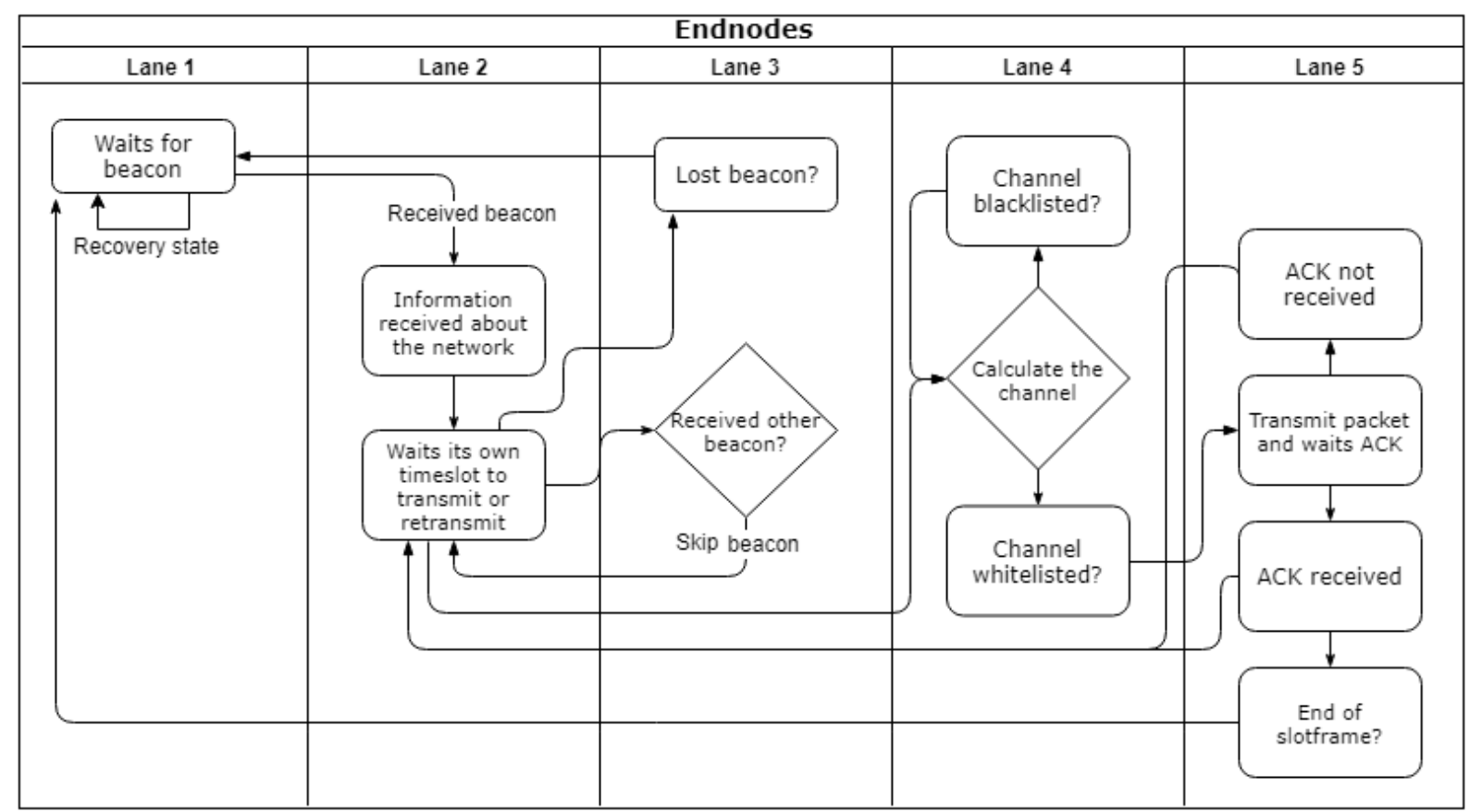

Figure 7: Diagram of the endnodes steps. 

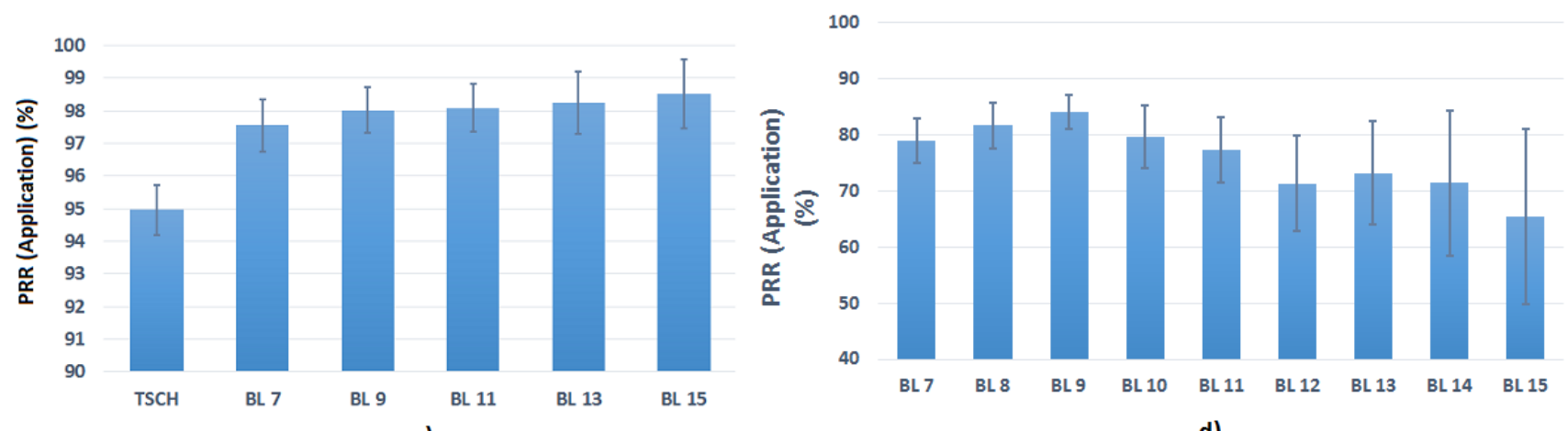

a)
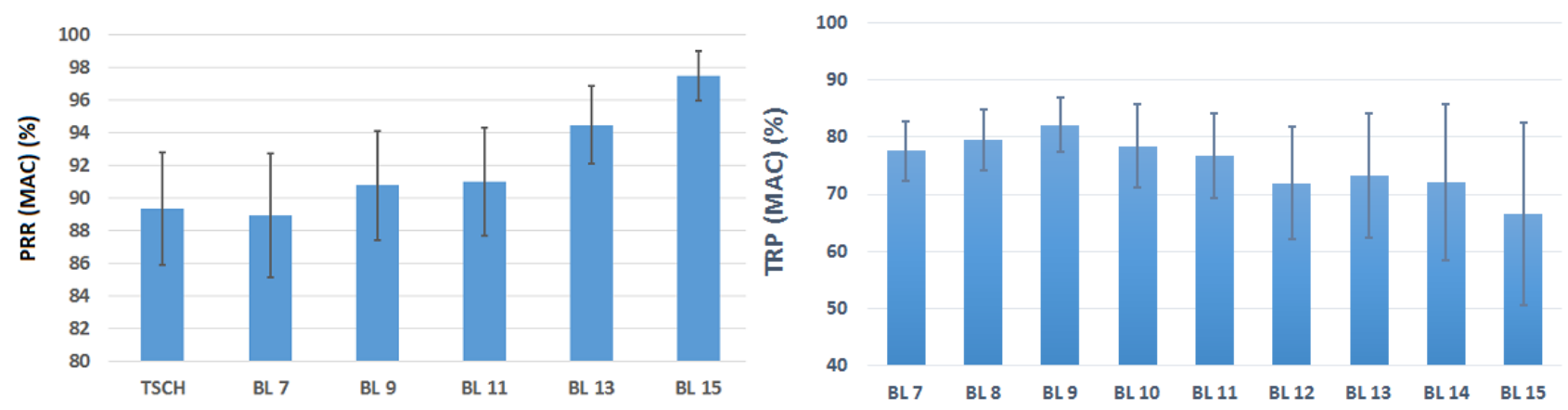

b)
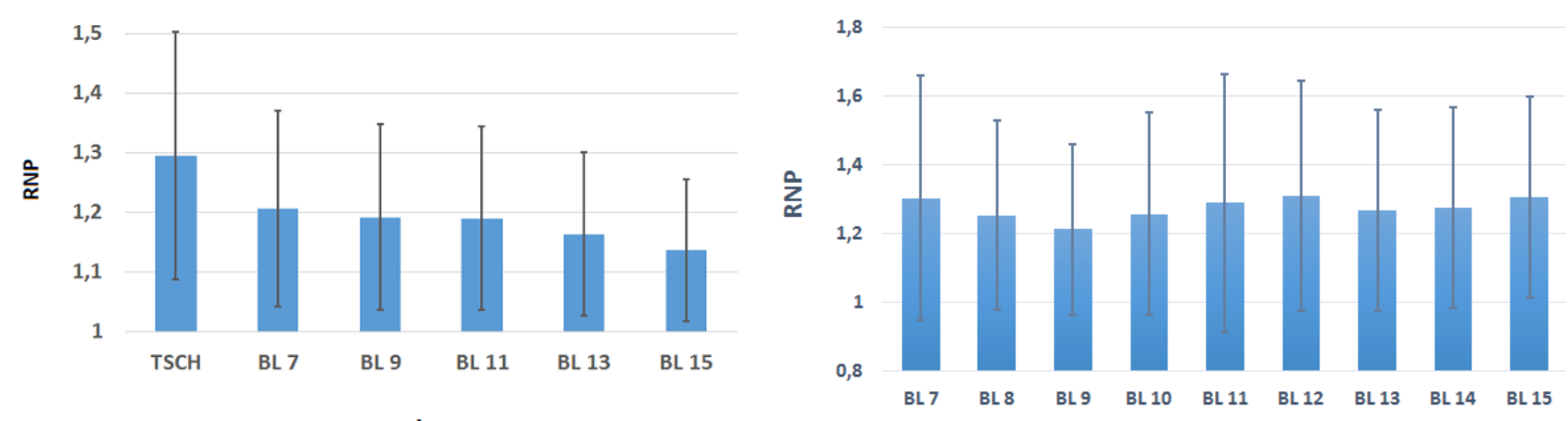

c)

f)

Figure 8: Results in star topology: a) PRR in Application layer. b) PRR in MAC layer. c) RNP, and results in tree topology: d) PRR in Application layer, e) PRR in MAC layer and f)RNP.

and as the blacklist increases in size, the quality of the star network also increases. This can be explained by the fact that, at each packet transmission, the node is on a different channel. By limiting the availability of channels, the nodes more frequently access the previous channels, allowing the estimator to check more precisely which channels are the best and the worst, blocking the latter. This way, the nodes will stay for longer term in channels with better quality. With 15 blocked channels, the nodes only switch to a better channel when the current channel presents lower performance.

In tree topology, depicted in Figure 5, the blacklist is randomly set at the beginning of the network. This approach can help nodes with simultaneous transmission to choose different channels, since the assigned channel in Eq. 1 is compared by AB-TSCH if it is blacklisted; if so, another channel is chosen until a whitelisted one is assigned. However, the use of different and non-adjacent channels with this approach is not fully guaranteed, since two nodes can choose the same channel after the calculation in Eq. 1. This issue is depicted in Figure 6 (b) in red for the links $10 \rightarrow 1$ and $12 \rightarrow 2$. It is necessary to develop methods that deal with the generation of local blacklists, and at the same time to program the nodes to transmit on different channels, and to avoid adjacent-channel interference. The 
Empirical cumulative distribution function (\%)

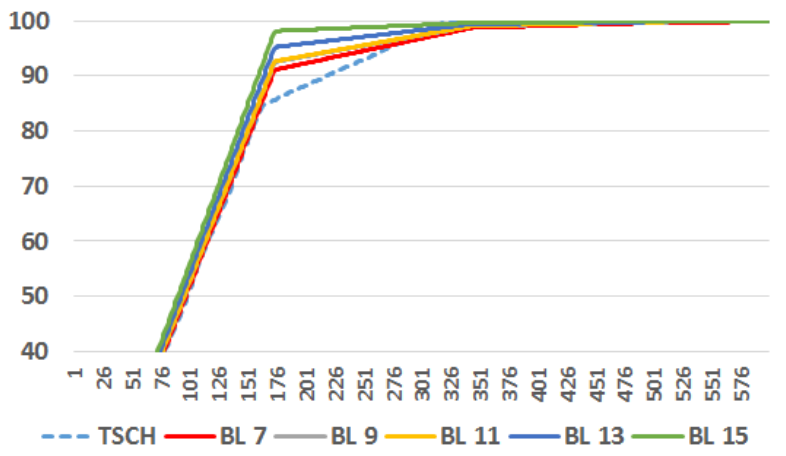

a)

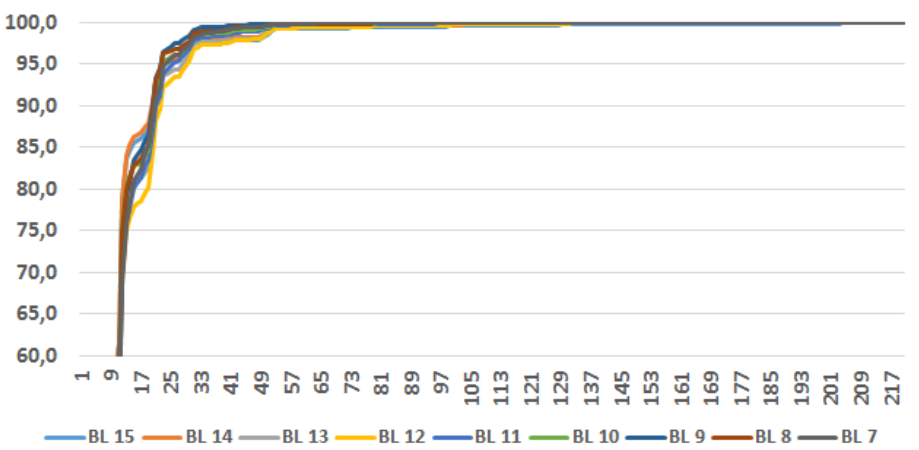

b)

Figure 9: Empirical cumulative distribution function of the delay in ms in star (a) and tree (b) topologies

problem with this kind of interference is illustrated in this Figure between the nodes $6 \rightarrow 1$ and $4 \rightarrow 2$, in channels 3 and 4 .

In Figure 6 (a), only the first timeslot is set for the sink node to transmit beacons, and each beacon is transmitted in a different channel. After receiving it, the node 1 sends a packet to the sink in the first channel $(2405 \mathrm{MHz})$, node 2 sends its packet in the third slot in frequency $2445 \mathrm{MHz}$, and so on. After all nodes sent their packets, the sink node transmit the second beacon in the second channel, and so on.

In Figure 6 (b), the sink node sends beacons in every channel the same way as in star network, and the cluster heads transmit beacons in the second slot using all the channels available. For transmission of beacons, there is no blacklist in this work, and the effect of bad quality channels in transmitting beacons is an issue to be researched.

Fig. 7 presents a simple diagram of the protocol AB-TSCH. If many beacons are lost in sequence (this threshold can be configured), the endnode enters a recovery state, in which it listens in each of the 16 channels during $\mathrm{k}$ slotframe periods, until a beacon is received.

\section{SIMULATION RESULTS}

Simulations were performed for networks with 16 endnodes with transmission power of $0 \mathrm{dBm}$, and transmission rate of one packet/s. The nodes were arranged in an area of $200 \times 200 \mathrm{~m}$, using 10 replications, and with the network running for two hours in two configurations, one for star network, and the other for tree network. At each replication, the position of the nodes was randomly assigned, with a maximum distance of $40 \sqrt{2} \mathrm{~m}$ from the sink node. In the case of tree network, there were one sink, two cluster heads, and 16 endnodes.

To guarantee a fair result, for each replication the same seed was used (seed is a parameter of the simulator) to evaluate both protocols in star network, TSCH and AB-TSCH, and different seeds were used in the different replications. Thus, both protocols were evaluated considering the nodes in the same position and with the same characteristics for the wireless channel during the replications. The second experiment with tree network used the same seed of star network, and was performed to evaluate the different sizes of blacklists in such topology with the AB-TSCH protocol.

In Figure 8 (a), PRR values are shown in the application layer for the TSCH protocol, and for AB-TSCH with blacklists (BL) of sizes 7, 9, 11, 13 and 15. The PRR in the application layer considers all aspects, including packet retransmissions and transmission failures of EBs. In Figure 8 (b), the PRR is shown in the MAC layer, which considers only the packets that were effectively transmitted, disregarding the dropped packets due to problems in reception of EBs or synchronization failures. This allows analyzing the quality of the links in only one direction, from the endnodes to the sink. In Figure 8 (c), the values relative to the RNP are shown, which is the average number of attempts made for each data packet generated in the network, regardless of reception. An attempt of transmission and one of retransmission were used, in case of not receiving the packet confirmation by the sink node, totaling two as the maximum value of the RNP.

When channel adaptation is used, which is a slow frequency hopping method, as in the 15-channel blacklist scenario, the results show a higher PRR than in the other configurations because the latter use fast frequency hopping. Simple TSCH behaves in a way that all channels are used without considering the use of a link quality estimator. Because the other configurations use this estimator, the result shows an improvement in the PRR, since the poor quality channels are blocked. Since the channel in the industrial environment undergoes many variations, the same channel that has been blocked can be removed from the blacklist after a certain period, depending on the variations of the environment. This issue was also considered in the development of the AB-TSCH, which for the context of this work, the reuse of blacklisted channels was done in a random way, however a smart reuse of those channels is required for future work.

Concerning the experiments with tree network, depicted in Figure 8 (d), the application layer with blacklists between blacklist 7 and 10 presented almost the same results, as well as in the MAC layer, depicted in Figure 8 (e). The relevant matter of this experiment is that, the larger the blacklist size for tree networks is, the smaller the number of packets received by the sink node is. This 
can be explained due to the interference of the same channels and adjacent channels caused by some nodes in simultaneous transmissions. With the limited channel diversity, many packets are lost or retransmitted. Different from the experiment in star network, the tree network presented almost the same results regarding the RNP for all the scenarios. This can be explained because this experiment was performed using only the AB-TSCH protocol, and there are no noticeable changes in each blacklist setting for the protocol. One factor to note in RNP is the high standard deviation in all blacklist scenarios, and because it behaved similarly to the TSCH in star network, with almost the same average number of retries made for each packet generated.

In Figure 9 (a), it is possible to see the empirical cumulative distribution function of the delay in star network, and in (b) for tree network. The delay is regarding the time interval between the reception of two consecutive packets for both protocols. Packets are generated at a rate of 1 packet/s at the application layer, but the delay to gain access to the channel and the failures in the transmissions cause variations in the time intervals between two packet receipts.

The graphic with the delay information only considers the packets actually received. The access delay is lower for the AB-TSCH protocol, since approximately $97 \%$ of the packets were delivered with a delay of less than $172 \mathrm{~ms}$. In the $\mathrm{TSCH}$, around $90 \%$ of the packets were delivered with a delay of less than $224 \mathrm{~ms}$. In the star network with 16 endnodes, the slotframe in the TSCH lasts 160 $\mathrm{ms}$, and in $\mathrm{AB}-\mathrm{TSCH}$, which considers the time interval used to transmit the beacon, lasts $170 \mathrm{~ms}$. In AB-TSCH, $99.5 \%$ of the packets were delivered with a delay of less than $340 \mathrm{~ms}$, which is equivalent to two slotframe periods.

When considering the empirical cumulative distribution function of the delay in tree network in Figure 9 (b), since this experiment was performed using the same protocol, but with different sizes of blacklist, the results showed almost the same values for all the sizes of blacklists. If compared to the star topology, the tree topology showed better performance when, for example, $98 \%$ of the packets were delivered with a delay of less than $40 \mathrm{~ms}$.

\section{CONCLUSIONS AND FUTURE WORK}

This article presents a TSCH-based protocol called $\mathrm{AB}-\mathrm{TSCH}$, which uses the EBs approach and dynamic configuration of channel blacklists through real-time link quality estimation in star and tree topology networks. The protocol was compared to the TSCH with simple configuration in star topology, and the AB-TSCH was evaluated with varying sizes of blacklists in both topologies. AB-TSCH with maximum blacklist (15 channels) has overcome the other configurations, since the node remains using a good channel for a longer term, and only performs a channel change when the channel in use becomes worse. This approach performed well, because the quality of the links was continuously monitored.

The experiment with tree network demonstrated the need for a correct channel establishment so that simultaneous transmissions avoid using similar or adjacent channels. It also showed that the larger the channels blacklist, the lower the diversity, and the lower the network capacity. There must be a limit of the blacklist size that ensures good quality and high throughput in communications, and this limit needs to be more investigated.

As future work, networks with varying node configurations will be analyzed, such as the number of retransmissions and the packet transmission rate, including an improvement of the protocol when considering tree topologies, which is more challenging than the star one due to the possibility of simultaneous transmissions.

\section{ACKNOWLEDGMENTS}

The authors are grateful for the support of COPELE-UFCG, the Institute for Advanced Studies in Communications (Iecom), the National Council for Scientific and Technological Development (CNPq), the Coordination for the Improvement of Higher Education Personnel (Capes), the Postgraduate Program in Electrical Engineering at the Federal University of Bahia, the EM-SMART2 project of the Erasmus Mundus Program, and the Radio Engineering Research Group (GIRA) of the Universidad Politécnica de Madrid (UPM).

\section{REFERENCES}

[1] Castalia. [n. d.]. Castalia Simulator. https://github.com/boulis/Castalia Access in: $30 / 05 / 2018$.

[2] Peng Du and George Roussos. 2012. Adaptive time slotted channel hopping for wireless sensor networks. In 2012 4th Computer Science and Electronic Engineering Conference (CEEC). IEEE, 29-34. https://doi.org/10.1109/CEEC.2012.6375374

[3] Simon Duquennoy, Beshr Al Nahas, Olaf Landsiedel, and Thomas Watteyne. 2015. Orchestra. In Proceedings of the 13th ACM Conference on Embedded Networked Sensor Systems - SenSys '15. ACM Press, New York, New York, USA, 337-350. https://doi.org/10.1145/2809695.2809714

[4] Pedro Henrique Gomes, Thomas Watteyne, and Bhaskar Krishnamachari. 2017. MABO-TSCH: Multihop and blacklist-based optimized time synchronized channel hopping. Transactions on Emerging Telecommunications Technologies (Aug. 2017), e3223. https://doi.org/10.1002/ett.3223

[5] Ruan D. Gomes, Marcelo S. Alencar, Diego V. Queiroz, Iguatemi E. Fonseca, and Cesar Benavente-Peces. 2017. Comparison between Channel Hopping and Channel Adaptation for Industrial Wireless Sensor Networks. In Proceedings of the 6th International Conference on Sensor Networks. SCITEPRESS - Science and Technology Publications, 87-98. https://doi.org/10.5220/0006206800870098

[6] Ruan D. Gomes, Diego V. Queiroz, Abel C.Lima Filho, Iguatemi E. Fonseca, and Marcelo S. Alencar. 2017. Real-time link quality estimation for industrial wireless sensor networks using dedicated nodes. Ad Hoc Networks 59 (May 2017), 116-133. https://doi.org/10.1016/j.adhoc.2017.02.007

[7] Ruan D. Gomes, Diego V. Queiroz, Iguatemi E. Fonseca, and Marcelo S. Alencar. 2017. A Simulation Model for Industrial Multi-Channel Wireless Sensor Networks. Journal of Communication and Information Systems 32, 1 (2017), 29-40. https: //doi.org/10.14209/jcis.2017.4

[8] IEEE. [n. d.]. IEEE Standard for Low-Rate Wireless Personal Area Networks (LR-WPANs). IEEE Std 802.15.4-2015 (Revision of IEEE Std 802.15.4-2011) (April 2016).

[9] Vasileios Kotsiou, Georgios Z. Papadopoulos, Periklis Chatzimisios, and Fabrice Theoleyre. 2017. LABeL: Link-based Adaptive BLacklisting Technique for $6 \mathrm{TiSCH}$ Wireless Industrial Networks. In Proceedings of the 20th ACM International Conference on Modelling, Analysis and Simulation of Wireless and Mobile Systems - MSWiM '17. ACM Press, New York, New York, USA, 25-33. https://doi.org/10.1145/3127540.3127541

[10] Diego V. Queiroz, Marcelo S. Alencar, Ruan D. Gomes, Iguatemi E. Fonseca, and Cesar Benavente-Peces. 2017. Survey and Systematic Mapping of Industrial Wireless Sensor Networks. Fournal of Network and Computer Applications 97, C (Nov. 2017), 96-125. https://doi.org/10.1016/j.jnca.2017.08.019

[11] E. Tanghe, W. Joseph, L. Verloock, L. Martens, H. Capoen, K. V. Herwegen, and W. Vantomme. 2008. The industrial indoor channel: large-scale and temporal fading at 900, 2400, and $5200 \mathrm{MHz}$. IEEE Transactions on Wireless Communications 7, 7 (2008), 2740-2751.

[12] Rasool Tavakoli, Majid Nabi, Twan Basten, and Kees Goossens. 2015. Enhanced Time-Slotted Channel Hopping in WSNs Using Non-intrusive Channel-Quality Estimation. In 2015 IEEE 12th International Conference on Mobile Ad Hoc and Sensor Systems. IEEE, 217-225. https://doi.org/10.1109/MASS.2015.48

[13] Dimitrios Zorbas, Vassilis Kotsiou, Fabrice Theoleyre, Georgios Z. Papadopoulos, and Christos Douligeris. 2018. LOST: Localized blacklisting aware scheduling algorithm for IEEE 802.15.4-TSCH networks. In 2018 Wireless Days (WD). IEEE, 110-115. https://doi.org/10.1109/WD.2018.8361705 